\title{
Sub-miliSievert ultralow-dose CT colonography with iterative model reconstruction technique
}

Lukas Lambert, Petr Ourednicek, Jan Briza, Walter Giepmans, Jiri Jahoda, Lukas Hruska, Jan Danes

Purpose: The purpose of this study was to evaluate technical and diagnostic performance of sub-miliSievert ultralow-dose (ULD) CT colonograpy (CTC) in the detection of colonic and extracolonic lesions. Materials and methods: CTC with standard dose (SD) and ULD acquisitions of 64 matched patients, half of them with colonic findings, were reconstructed with filtered back projection (FBP), hybrid (HIR) and iterative model reconstruction techniques (IMR). Image noise in six colonic segments, in the left psoas muscle and aorta were measured. Image quality of the left adrenal gland and of the colon in the endoscopic and $2 \mathrm{D}$ view was rated on a five point Likert scale by two observers, who also completed the reading of CTC for colonic and extracolonic findings. Results: The mean radiation dose estimate was $4.1 \pm 1.4 \mathrm{mSv}$ for SD and $0.86 \pm 0.17 \mathrm{mSv}$ for ULD for both positions $(p<0.0001)$. In ULD-IMR, SD-IMR and SD-HIR, the endoluminal noise was decreased in all colonic segments compared to SD-FBP $(p<0.001)$. There were 27 small $(6-9 \mathrm{~mm})$ and 17 large $(\geq 10 \mathrm{~mm})$ colonic lesions that were classified as sessile polyps $(n=38)$, flat lesions $(n=3)$, or as a mass $(n=3)$. Per patient sensitivity and specificity were 0.82 and 0.93 for ULD-FBP, 0.97 and 0.97 for ULD-HIR, 0.97 and 1.0 for ULD-IMR. Per polyp sensitivity was 0.84 for ULD-FBP, 0.98 for ULD-HIR, 0.98 for ULD-IMR. Significantly less extracolonic findings were detected in ULD-FBP and ULD-HIR, but in the E4 category by C-RADS (potentially important findings), the detection was similar. Conclusion: Both HIR and IMR are suitable for sub-miliSievert ULD CTC without sacrificing diagnostic performance of the study. 
1 Sub-miliSievert ultralow-dose CT colonography with iterative model reconstruction

2 technique

3 Type of manuscript: original research article

4 Lukas Lambert $^{1}$, Petr Ourednicek ${ }^{2}$, Jan Briza $^{3}$, Walter Giepmans ${ }^{4}$, Jiri Jahoda ${ }^{1}$, Lukas Hruska $^{2}$,

5 Jan Danes ${ }^{1}$

$6{ }^{1}$ Department of Radiology, First Faculty of Medicine, Charles University in Prague and General

7 University Hospital in Prague

$8{ }^{2}$ Department of Imaging Methods, St. Anne's University Hospital in Brno

$9{ }^{3}$ First Department of Surgery, First Faculty of Medicine, Charles University in Prague and

10 General University Hospital in Prague

$11{ }^{4}$ Philips Healthcare, Best, The Netherlands

12 Running head: Sub-miliSievert ultralow-dose CTC

\section{Corresponding author}

14 Lukas Lambert, M.D. M.S.C.S. Ph.D., Department of Radiology, General University Hospital in

15 Prague, U Nemocnice 2, 12808 Prague 2, Czech Republic, tel.: +420 224962232, fax.: +420

16 224963048, e-mail: lambert.lukas@gmail.com 


\section{Abstract}

18 Purpose: The purpose of this study was to evaluate technical and diagnostic performance of submiliSievert ultralow-dose (ULD) CT colonograpy (CTC) in the detection of colonic and

20 extracolonic lesions.

21 Materials and methods: CTC with standard dose (SD) and ULD acquisitions of 64 matched 22 patients, half of them with colonic findings, were reconstructed with filtered back projection 23 (FBP), hybrid (HIR) and iterative model reconstruction techniques (IMR). Image noise in six 24 colonic segments, in the left psoas muscle and aorta were measured. Image quality of the left 25 adrenal gland and of the colon in the endoscopic and 2D view was rated on a five point Likert 26 scale by two observers, who also completed the reading of CTC for colonic and extracolonic 27 findings.

28 Results: The mean radiation dose estimate was $4.1 \pm 1.4 \mathrm{mSv}$ for $\mathrm{SD}$ and $0.86 \pm 0.17 \mathrm{mSv}$ for ULD 29 for both positions ( $\mathrm{p}<0.0001)$. In ULD-IMR, SD-IMR and SD-HIR, the endoluminal noise was decreased in all colonic segments compared to SD-FBP $(\mathrm{p}<0.001)$. There were 27 small $(6-9 \mathrm{~mm})$ and 17 large $(\geq 10 \mathrm{~mm})$ colonic lesions that were classified as sessile polyps $(\mathrm{n}=38)$, flat lesions

$32(n=3)$, or as a mass $(n=3)$. Per patient sensitivity and specificity were 0.82 and 0.93 for ULD33 FBP, 0.97 and 0.97 for ULD-HIR, 0.97 and 1.0 for ULD-IMR. Per polyp sensitivity was 0.84 for 34 ULD-FBP, 0.98 for ULD-HIR, 0.98 for ULD-IMR. Significantly less extracolonic findings were 35 detected in ULD-FBP and ULD-HIR, but in the E4 category by C-RADS (potentially important 36 findings), the detection was similar.

37 Conclusion: Both HIR and IMR are suitable for sub-miliSievert ULD CTC without sacrificing 38 diagnostic performance of the study. 


\section{Introduction}

Over the last decade, we have witnessed substantial improvements in the iterative reconstruction technique that ultimately resulted in introduction of iterative model reconstruction (IMR) technique into practice by major CT vendors (Löve et al., 2013). Compared to filtered back projection (FBP), which is a standard single-pass analytical method for producing CT images from attenuation coefficients measured by a CT detector assuming monoenergetic x-ray beam, ideal physics and geometry of the system, iterative reconstruction techniques use a multipass algorithm that additionally models real system geometry, x-ray beam statistics (different attenuation of parts of the polyenergetic x-ray spectrum), and encourages desirable image properties (smoothness, edges) (Mehta et al., 2013).

Unlike previous generations of iterative reconstruction techniques (statistical, hybrid) model-based solutions approach reconstruction as an iterative optimization process to find the "best fit" image to the acquired data, while penalizing the noise, through the use of data statistics, image statistics, and system models (Mehta et al., 2013). This results in greater reduction of the image noise, suppression of artifacts, improved spatial and low contrast resolution with greater scope for dose reduction while maintaining diagnostic image quality (Hara et al., 2009; Mehta et al., 2013; Lambert et al., 2015b).

Even though the technical performance of IMR has been validated early, the evaluation of diagnostic performance in specific applications unfolded gradually (McCollough et al., 2009; Flicek et al., 2010; Lambert et al., 2015b). In CT colonography (CTC), decreasing the radiation dose is even more important. Patients undergo CTC not only after incomplete optical colonoscopy (OC) or if colonic cancer is suspected, but also for primary screening (Brenner \& Georgsson, 2005). Apart from reimbursement, radiation burden from CTC screening may be a concern because healthy individuals are exposed to radiation which is a weak carcinogen itself (Albert, 2013). So far, several papers on the technical performance of sub-miliSievert ultralowdose (ULD) CTC have been published and there is limited information about its diagnostic performance and its improvement by IMR (Lambert et al., 2015a,b; Nagata et al., 2015; Lubner et al., 2015).

In this study, we compared the diagnostic performance of sub-miliSievert ULD CTC with standard dose (SD) CTC reconstructed with FBP, hybrid iterative reconstruction (HIR) and IMR techniques in the detection of colonic and extracolonic lesions. 
70

71

72

73

74

75

76

77

78

79

80

81

82

83

84

85

86

87

88

89

90

91

92

93

94

95

96

97

98

99

\section{Material \& Methods}

This prospective HIPAA compliant IRB approved study (reference number 1751/13 S/IV) was conducted in agreement with the Declaration of Helsinki and all patients signed an informed consent.

Between January 2014 and November 2014, 174 patients underwent CTC with two acquisitions per position where the standard dose was split in the proportion of 1:5. In 32 of them at least one colonic lesion (colonic polyp $\geq 6 \mathrm{~mm}$ in diameter or a colonic mass) was found. From the rest, another 32 age-, BMI-, and gender-matched patients with no colonic lesions were selected. The age of the patients was $67 \pm 12$ years and $42 \%$ were males.

CTC was performed after cathartic preparation with $200 \mathrm{~mL}$ of $40 \%$ magnesium sulfate in the evening, stool tagging with 250mL 2.1\% barium (Micropaque CT, GUERBET, Roissy, France) in the morning, noon and afternoon and dietary restriction on the day prior to the examination. Spasmolytic (butylscopolamine, Buscopan ${ }^{\circledR}$, Boehringer Ingelheim, Germany) was administered to $94 \%$ of patients (4 patients had contraindications). Insufflation of the colon by carbon dioxide was achieved by using a dedicated insufflator $\left(\mathrm{PROTOCO}_{2} \mathrm{~L}\right.$, Bracco Diagnostics Inc., NJ, USA).

The patients were scanned twice, both in the supine and prone positions at end-inspiration on iCT Brilliance CT scanner (Philips Healthcare, Best, The Netherlands) with the following parameters in both positions: peak tube voltage $120 \mathrm{kV}$, planned tube time current product $50 \mathrm{mAs}$ for SD acquisition and 10mAs for ULD acquisition, detector collimation $128 \mathrm{x} 0.625 \mathrm{~mm}$, rotation time $0.5 \mathrm{~s}$, pitch 0.601 , and with current modulation (DoseRight ${ }^{\mathrm{TM}}$ ). The images were reconstructed in $0.9 \mathrm{~mm}$ sections using a soft reconstruction kernel (filter A) for FBP and HIR $\left(\right.$ iDose $\left.^{4}\right)$ set on the maximum level (level 6), and a routine body IMR level 2 (level 1 = weak, level 3 =strong). IMR is currently the latest commercially available generation of iterative reconstruction by the scanner manufacturer. All pairs of datasets were anonymized and transferred to a client workstation (Philips Intellispace Portal) with a dedicated CT colonography package and computer aided detection (CAD).

The images were reviewed by two independent readers with experience in reading CTC $(>1300$ and $>800$ cases, respectively). The blinded studies were reviewed in a random order during a span of 6 months to minimize recall bias (Pickhardt et al., 2012). Colonic findings were 
100 primarily assessed in endoluminal or fillet view with CAD as a concurrent reader (Choi et al.,

101 2011). A difference in identification of polyps between the two observers that occurred in five

102 patients was resolved by consensus. The size of the polyps was measured in the endoluminal

103 view and diminutive polyps ( $<6 \mathrm{~mm}$ in diameter) were not reported (Pickhardt et al., 2008a).

104 Both readers assessed image quality (IQ) of the colon in virtual endoscopic / fillet view

105 and in 2D view (thin sections), and of the left adrenal gland (5mm section thickness) on a five

106 point Likert scale ( $1=$ excellent, $5=$ unevaluable), and reported colonic and extracolonic lesions

107 according to daily practice and C-RADS classification (Zalis et al., 2005; Lambert et al.,

108 2015a,b). The preferred endoluminal rendering threshold (HU value above which a voxel is

109 rendered as colonic wall instead of intraluminal air) and the number of CAD marks were

110 recorded as well. Image noise expressed as a standard deviation of Hounsfield density was

111 measured by a technologist in identical parts of all six colonic segments (rectum, sigmoid,

112 descending, transverse, ascending colon and cecum), in the aorta and in the left psoas muscle at

113 the level of the fifth lumbar vertebra using a fixed region of interest.

114 The radiation dose was estimated from the dose length product multiplied by a weighting 115 factor of $15 \mu \mathrm{Sv} / \mathrm{mGy} \cdot \mathrm{cm}$ (Christner, Kofler \& McCollough, 2010) and in seven patients also 116 using ImPACT CT Patient Dosimetry Calculator (ImPACT, London, UK).

117 Statistical evaluation was performed in Prism (Graphpad Software Inc., La Jolla, CA, 118 USA) and R (The R Foundation for Statistical Computing, Vienna, Austria). We used the

119 Friedman test with Dunns post hoc tests to compare the acquisitions and reconstruction

120 algorithms. An exact binomial test was used to compare sensitivity and specificity. Interobserver

121 agreement was expressed as Goodman and Kruskal's gamma statistics. SD acquisition served as

122 the reference standard. A P-value below 0.05 was considered significant.

\section{Results}

124 The average BMI of patients was $26.6 \pm 4.8 \mathrm{~kg} / \mathrm{cm}^{2}$ and the mean radiation dose estimate 125 was $4.1 \pm 1.4 \mathrm{mSv}$ for SD and $0.86 \pm 0.17 \mathrm{mSv}(\mathrm{p}<0.0001)$ for ULD for both positions.

126 The endoluminal noise per colonic segment, image quality in the virtual endoscopic and

127 2D view, preferred endoluminal rendering threshold, and clinical images are shown in Figs. 1-4.

128 There were 27 small $(6-9 \mathrm{~mm})$ and 17 large $(\geq 10 \mathrm{~mm})$ colonic lesions that were classified as

129 sessile polyps $(n=38)$, flat lesions $(n=3)$, or as a mass $(n=3)$. The detection rate was lower for 
130 ULD-FBP compared to other reconstruction techniques $(\mathrm{p}=0.020)$ and there were also more false 131 positive results ( $p=0.011$, Fig. 5). Per patient sensitivity and specificity were 0.82 (95\%CI $0.66-$ $1320.93, \mathrm{p}=0.031)$ and $0.93(0.76-0.99, \mathrm{p}=0.5)$ for ULD-FBP, $0.97(95 \% \mathrm{CI} 0.83-1.0, \mathrm{p}=1.0)$ and $1330.97(0.80-1.0, \mathrm{p}=1.0)$ for ULD-HIR, $0.97(95 \%$ CI $0.83-1.0, \mathrm{p}=1.0)$ and $1.0(0.85-1.0$, $134 \mathrm{p}=1.0)$ for ULD-IMR. Per polyp sensitivity was $0.84(0.64-0.93, \mathrm{p}=0.016)$ for ULD-FBP, 0.98 $135(0.88-1.0, \mathrm{p}=1.0)$ for ULD-HIR, and $0.98(0.88-1.0, \mathrm{p}=1.0)$ for ULD-IMR. In the local 136 colonoscopy database, we found that 23 lesions in 16 patients were verified, the rest of the 137 patients underwent colonoscopy elsewhere, or was scheduled for follow-up, or the findings were 138 deemed unimportant by the physician.

There was no significant difference in the size and volume of polyps among all reconstruction techniques ( $\mathrm{p}=0.077$ for size, $\mathrm{p}=0.49$ for volume). There were significantly less extracolonic findings detected in ULD-FBP and ULD-HIR, but in the E4 category (potentially

142 important findings), the detection was similar (Fig. 6). The image noise in the aorta and in the left 143 psoas muscle and the image quality of the left adrenal gland are shown in Fig. 6. The 144 approximate reconstruction times were 40s per position for FBP, 60s for HIR, and 80s for IMR.

The interobserver agreement for image quality of the virtual endoscopic, 2D view, and the 146 left adrenal gland was $0.91,0.90$, and 0.83 , respectively.

\section{Discussion}

Model based reconstruction is now commercially available in CT scanners of major vendors, who promise up to $80 \%$ reduction of the radiation dose while maintaining image noise and resolution (Mehta et al., 2013; Löve et al., 2013). CTC is one of the applications where reducing the radiation dose is of major importance. It is increasingly available and used for screening of asymptomatic individuals (Yee, 2013; Pickhardt, 2015). The estimated benefit to risk ratio of CTC can be increased in direct proportion to dose reduction provided that polyp detection and discrimination among true polyps and polyp mimics remains unchanged. In case of submiliSievert ULD-CTC it can rise up to 209:1 (de Gonzalez et al., 2011).

In order to reduce radiation dose from CTC, several optimization strategies must be employed simultaneously (Chang \& Yee, 2013). The radiation dose from CTC followed the same descending trend as other CT examinations. The majority of CTC studies in patients published in 
160 addressing the radiation dose. First attempts to reduce the radiation dose in images reconstructed

161 with FBP with a special noise-reducing filters resulted in dose estimates close to $2 \mathrm{mSv}$ per study

162 (Iannaccone et al., 2003; Cohnen et al., 2004). The iterative reconstruction technique that has

163 been tested for CTC in the last five years, could decrease the dose even further, close to or even

164 below $1 \mathrm{mSv}$ for both positions altogether (Lambert et al., 2015a,b; Lubner et al., 2015). The

165 introduction of size specific dose estimate (SSDE), which was not used in this study is bound to

166 even decrease the dose estimate in our slightly overweight patients but not on the absorbed dose

167 per se (Christner et al., 2012; Lubner et al., 2015).

168 Although most studies in ULD-CTC reported unchanged polyp conspicuity that is closely

169 related to their detection by both the human reader and CAD, it is also the ability to discriminate

170 between polyps and polyp mimics (poorly tagged stool residuals, folds, inverted diverticula)

171 especially in less distended segments, which makes an excellent reader and excellent CAD

172 (Fisichella et al., 2010; Lefere \& Gryspeerdt, 2011; Pickhardt \& Kim, 2013). Unfortunately, the

173 homogeneity of small polyps is difficult to measure because of the effect of partial volume

174 averaging. Instead, surrogate parameters such as image noise in a different (larger) structure and

175 subjective assessment of IQ are used to assess technical performance of the study. At the

176 diagnostic level, this is reflected in false positive rather than false negative findings and in the

177 fact that the performance of polyp detection declines more than the number of CAD marks, as

178 also shown in this study (Näppi \& Yoshida, 2007). In ULD-FBP, the IQ is reduced below an

179 acceptable level which results in decreased sensitivity and specificity compared to HIR-ULD and

180 IMR-ULD acquisitions where diagnostic performance approaches that of SD. The substantially

181 decreased IQ of ULD-FBP is also reflected in its technical performance by increased image noise

182 and poor ratings of IQ by the readers especially in the rectum and sigmoid colon. The perceived

183 IQ in the endoluminal view can be to some extent improved by increasing the endoluminal

184 rendering threshold, but this in turn results in a decreased size of the lesions that become less

185 conspicuous (Lambert et al., 2015b).

186 The polyp size is an important biomarker of its position in the adenoma - carcinoma 187 sequence (Summers, 2010). In this study, the polyp size and volume among SD and ULD did not 188 vary significantly which means that HIR or IMR can be safely introduced without any correction 189 of these measurements. There are other, more important variables influencing polyp size such as 190 distension, endoluminal rendering threshold and the viewing window (Taylor et al., 2006;

191 Summers, 2010). 
192

193

194

195

196

197

198

199

200

201

202

203

204

205

206

207

208

209

210

211

212

213

214

215

216

217

218

219

220

The detection of extracolonic pathology is considered one of the advantages of CTC over OC (Pickhardt et al., 2008b; Badiani et al., 2013). In this study, ULD examinations reconstructed with either FBP or HIR resulted in significantly lower detection of clinically unimportant findings (E2 category by C-RADS), which obviously had no clinical importance, and the number of potentially important findings (E4 by C-RADS) remained stable. In ULD-FBP, a lesion otherwise classified as "clinically unimportant" (E2) may turn into a "likely unimportant finding, incompletely characterized" due to limited visualization of its internal structure or increased density by excessive noise. This may result in the need of unnecessary workup and increased cost.

It has already been reported, that decreasing the radiation dose from CTC by half does not have any effect on polyp detection and lesion conspicuity, notably when iterative reconstruction is used, which makes the SD study acquired with $83 \%$ of the original dose (compared to the previous acquisition protocol) a valid standard of reference (Flicek et al., 2010; Lubner et al., 2015). The cumulative dose (about $5 \mathrm{mSv}$ ) was in line with what is currently done in the majority of institutions practicing CTC (de Gonzalez et al., 2011; Albert, 2013). The use of an additional ULD scan to assess its performance has already been reported in the literature (Lubner et al., 2015).

This study has several limitations. Firstly, the image appearance of different reconstruction techniques used in this study is well recognizable and therefore blinding of the studies may not have been effective enough. Secondly, we examined a solution by a single vendor, but other studies suggest that sub-miliSievert CTC is feasible with other CT scanners as well (Flicek et al., 2010; Lubner et al., 2015). Although the studies were reviewed in random order and with sufficient washout period, we cannot entirely exclude the effect of recall bias (Pickhardt et al., 2012). Since the predictive values are dependent on the prevalence of the disease according to Bayes' theorem, this study did not evaluate the negative and positive predictive values, due to the low prevalence of disease in the study population (18\%). Because we use barium tagging that results in inhomogeneous opacification of intraluminal fluid, electronic cleansing is not used and therefore it was not tested. As the standard of reference, standard dose CTC was used, which has performance comparable to OC (Pickhardt et al., 2003). 
221 In conclusion, this study showed that both hybrid and iterative model reconstruction

222 techniques are suitable for sub-miliSievert ultralow-dose CT colonography without sacrificing 223 the diagnostic performance of the study. 
224

225

226

227

228

\section{References}

Albert JM. 2013. Radiation risk from CT: implications for cancer screening. Am J Roentgenol 201:W81-87.

Badiani S, Tomas-Hernandez S, Karandikar S, Roy-Choudhury S. 2013. Extracolonic findings (ECF) on CT colonography (CTC) in patients presenting with colorectal symptoms. Acta Radiol:ACR486371.

Brenner DJ, Georgsson MA. 2005. Mass Screening With CT Colonography: Should the Radiation Exposure Be of Concern? Gastroenterology 129:328-337.

Cohnen M, Vogt C, Beck A, Andersen K, Heinen W, vom Dahl S, Aurich V, Haeussinger D, Moedder U. 2004. Feasibility of MDCT Colonography in ultra-low-dose technique in the detection of colorectal lesions: comparison with high-resolution video colonoscopy. Am J Roentgenol 183:1355-1359.

Fisichella VA, Båth M, Johnsson ÅA, Jäderling F, Bergsten T, Persson U, Mellingen K, Hellström M. 2010. Evaluation of image quality and lesion perception by human readers on 3D CT colonography: comparison of standard and low radiation dose. Eur Radiol 20:630-639.

Flicek KT, Hara AK, Silva AC, Wu Q, Peter MB, Johnson CD. 2010. Reducing the radiation dose for CT colonography using adaptive statistical iterative reconstruction: A pilot study. Am J Roentgenol 195:126-131.

De Gonzalez AB, Kim KP, Knudsen AB, Lansdorp-Vogelaar I, Rutter CM, Smith-Bindman R, Yee J, Kuntz KM, van Ballegooijen M, Zauber AG, Berg CD. 2011. Radiation-related cancer risks from CT colonography screening: a risk-benefit analysis. Am J Roentgenol 196:816-823. 
246 Hara AK, Paden RG, Silva AC, Kujak JL, Lawder HJ, Pavlicek W. 2009. Iterative reconstruction

247 technique for reducing body radiation dose at CT: feasibility study. Am J Roentgenol

$248 \quad 193: 764-771$.

249 Chang KJ, Yee J. 2013. Dose reduction methods for CT colonography. Abdominal Imaging

$250 \quad 38: 224-232$.

251 Choi J-I, Kim SH, Kim SH, Park H-S, Lee JM, Lee JY, Han JK, Choi BI. 2011. Comparison of accuracy and time-efficiency of CT colonography between conventional and panoramic 3D interpretation methods: An anthropomorphic phantom study. Eur J Radiol 80:e68-

255 Christner JA, Braun NN, Jacobsen MC, Carter RE, Kofler JM, McCollough CH. 2012. Sizespecific Dose Estimates for Adult Patients at CT of the Torso. Radiology 265:841-847.

Christner JA, Kofler JM, McCollough CH. 2010. Estimating Effective Dose for CT Using DoseLength Product Compared With Using Organ Doses: Consequences of Adopting International Commission on Radiological Protection Publication 103 or Dual-Energy

Iannaccone R, Laghi A, Catalano C, Brink JA, Mangiapane F, Trenna S, Piacentini F, Passariello R. 2003. Detection of Colorectal Lesions: Lower-Dose Multi-Detector Row Helical CT Colonography Compared with Conventional Colonoscopy 1. Radiology 229:775-781.

Lambert L, Danes J, Jahoda J, Masek M, Lisy J, Ourednicek P. 2015a. Submilisievert ultralowdose CT colonography using iterative reconstruction technique: a feasibility study. Acta Radiol 56:517-525.

267 Lambert L, Ourednicek P, Jahoda J, Lambertova A, Danes J. 2015b. Model-based vs hybrid Radiol 88:20140667. 
270 Lefere P, Gryspeerdt S. 2011. CT colonography: avoiding traps and pitfalls. Insights Imaging

$271 \quad 2: 57-68$

272 Löve A, Olsson M-L, Siemund R, Stålhammar F, Björkman-Burtscher IM, Söderberg M. 2013.

273 Six iterative reconstruction algorithms in brain CT: a phantom study on image quality at

274 different radiation dose levels. Br J Radiol 86:20130388.

275 Lubner MG, Pooler BD, Kitchin DR, Tang J, Li K, Kim DH, Del Rio AM, Chen G-H, Pickhardt

276 PJ. 2015. Sub-milliSievert (sub-mSv) CT colonography: a prospective comparison of

277 image quality and polyp conspicuity at reduced-dose versus standard-dose imaging. Eur

$278 \quad$ Radiol 25:2089-2102.

279 McCollough CH, Primak AN, Braun N, Kofler J, Yu L, Christner J. 2009. Strategies for Reducing

280 Radiation Dose in CT. Radiol Clin North Am 47:27-40.

281 Mehta D, Thompson R, Morton T, Dhanantwari A, Shefer E. 2013. Iterative model

282 reconstruction: simultaneously lowered computed tomography radiation dose and

283 improved image quality. Med Phys Int J 2:147-55.

284 Nagata K, Fujiwara M, Kanazawa H, Mogi T, Iida N, Mitsushima T, Lefor AT, Sugimoto H.

285

2015. Evaluation of dose reduction and image quality in CT colonography: comparison of

286

low-dose CT with iterative reconstruction and routine-dose $\mathrm{CT}$ with filtered back

287 projection. Eur Radiol 25:221-229.

288 Näppi J, Yoshida H. 2007. Fully automated three-dimensional detection of polyps in fecal289 tagging CT colonography. Acad Radiol 14:287-300.

290 Pickhardt PJ, Choi JR, Hwang I, Butler JA, Puckett ML, Hildebrandt HA, Wong RK, Nugent PA, 291 Mysliwiec PA, Schindler WR. 2003. Computed tomographic virtual colonoscopy to screen for colorectal neoplasia in asymptomatic adults. N Engl J Med 349:2191-2200. 
293 Pickhardt PJ, Hassan C, Laghi A, Zullo A, Kim DH, Iafrate F, Morini S. 2008a. Small and

294 diminutive polyps detected at screening CT colonography: a decision analysis for referral 295 to colonoscopy. Am J Roentgenol 190:136-144.

296 Pickhardt PJ, Hanson ME, Vanness DJ, Lo JY, Kim DH, Taylor AJ, Winter TC, Hinshaw JL.

297 2008b. Unsuspected Extracolonic Findings at Screening CT Colonography: Clinical and 298 Economic Impact1. Radiology 249:151-159.

299 Pickhardt PJ, Lubner MG, Kim DH, Tang J, Ruma JA, del Rio AM, Chen G-H. 2012. Abdominal 300 CT With Model-Based Iterative Reconstruction (MBIR): Initial Results of a Prospective 301 Trial Comparing Ultralow-Dose With Standard-Dose Imaging. Am J Roentgenol $302 \quad 199: 1266-1274$.

303 Pickhardt PJ. 2015. CT Colonography for Population Screening: Ready for Prime Time? Dig Dis $304 \quad$ Sci 60:647-659.

305 Pickhardt PJ, Kim DH. 2013. CT Colonography: Pitfalls in Interpretation. Radiol Clin North Am $306 \quad 51: 69-88$.

307 Summers RM. 2010. Polyp size measurement at CT colonography: what do we know and what 308 do we need to know? Radiology 255:707-720.

309 Taylor S, Slater A, Honeyfield L, Burling D, Halligan S. 2006. CT colonography: effect of 310 colonic distension on polyp measurement accuracy and agreement-in vitro study. Acad $311 \quad$ Radiol 13:850-859.

312 Yee J. 2013. Advances in CT Colonography for Colorectal Cancer Screening and Diagnosis. J $313 \quad$ Cancer 4:200-209.

314 Zalis ME, Barish MA, Choi JR, Dachman AH, Fenlon HM, Ferrucci JT, Glick SN, Laghi A, 315 Macari M, McFarland EG, Morrin MM, Pickhardt PJ, Soto J, Yee J. 2005. CT 316 Colonography Reporting and Data System: A Consensus Proposal. Radiology 236:3-9. 
A

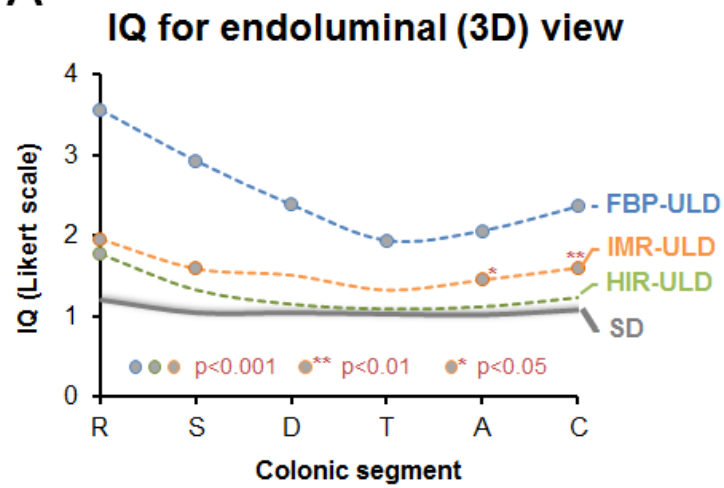

C

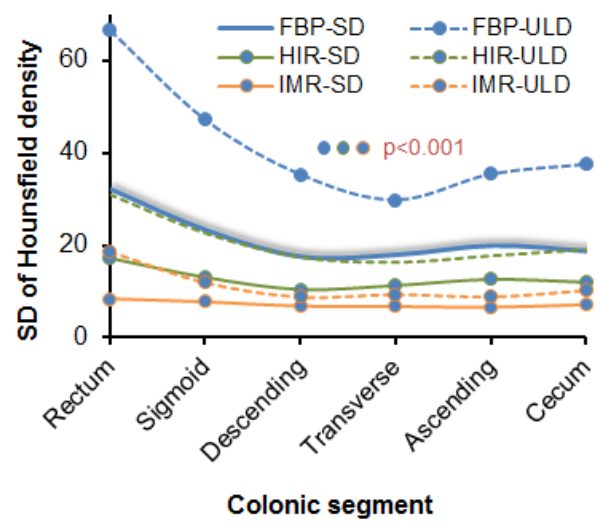

B

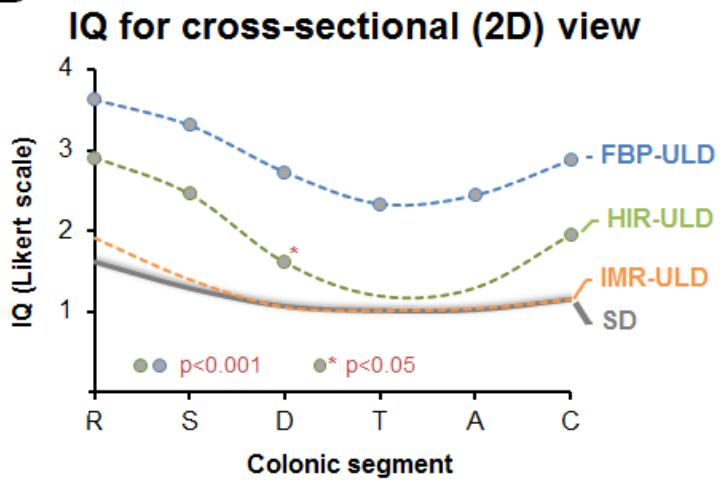

D Preferred reading threshold

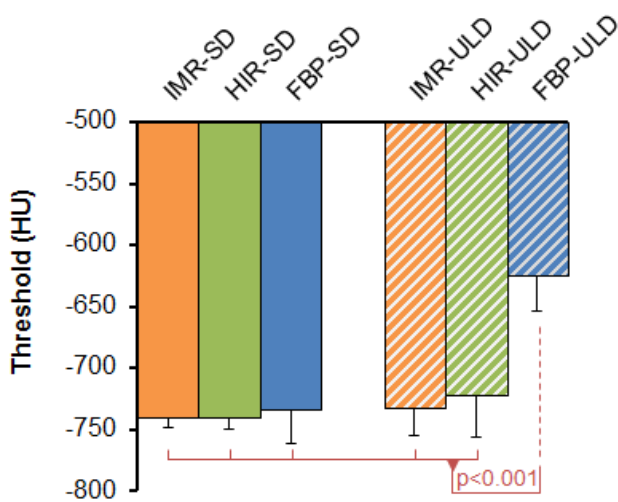

318 Fig. 1. Image quality (IQ) ratings for endoluminal (A) and cross-sectional (B) view for each 319 colonic segment ( $1=$ excellent, $5=$ unevaluable) compared to the standard dose (SD) acquisitions 320 (grey), which are represented as average from FBP-SD, HIR-SD, IMR-SD, show superiority of

321 both iterative reconstruction algorithms compared to FPB in ultralow-dose CT colonography.

322 Endoluminal noise measured as standard deviation of Hounsfield density in colonic lumen is

323 suppressed with IMR-ULD, IMR-SD, and HIR-SD compared to FBP-SD (C). Statistical

324 difference per segment is marked by circles. The preferred endoluminal rendering threshold, i.e.

325 Hounsfield density that discriminates voxels representing intraluminal air from the colonic wall

326 was significantly decreased in FBP-ULD indicating the need to suppress excessive noise (D).

$327 \mathrm{FBP}=$ filtered back projection, $\mathrm{HIR}=$ hybrid iterative reconstruction, $\mathrm{IMR}=$ iterative model

328 reconstruction technique, $\mathrm{SD}=$ standard dose, $\mathrm{ULD}=$ ultralow-dose. 


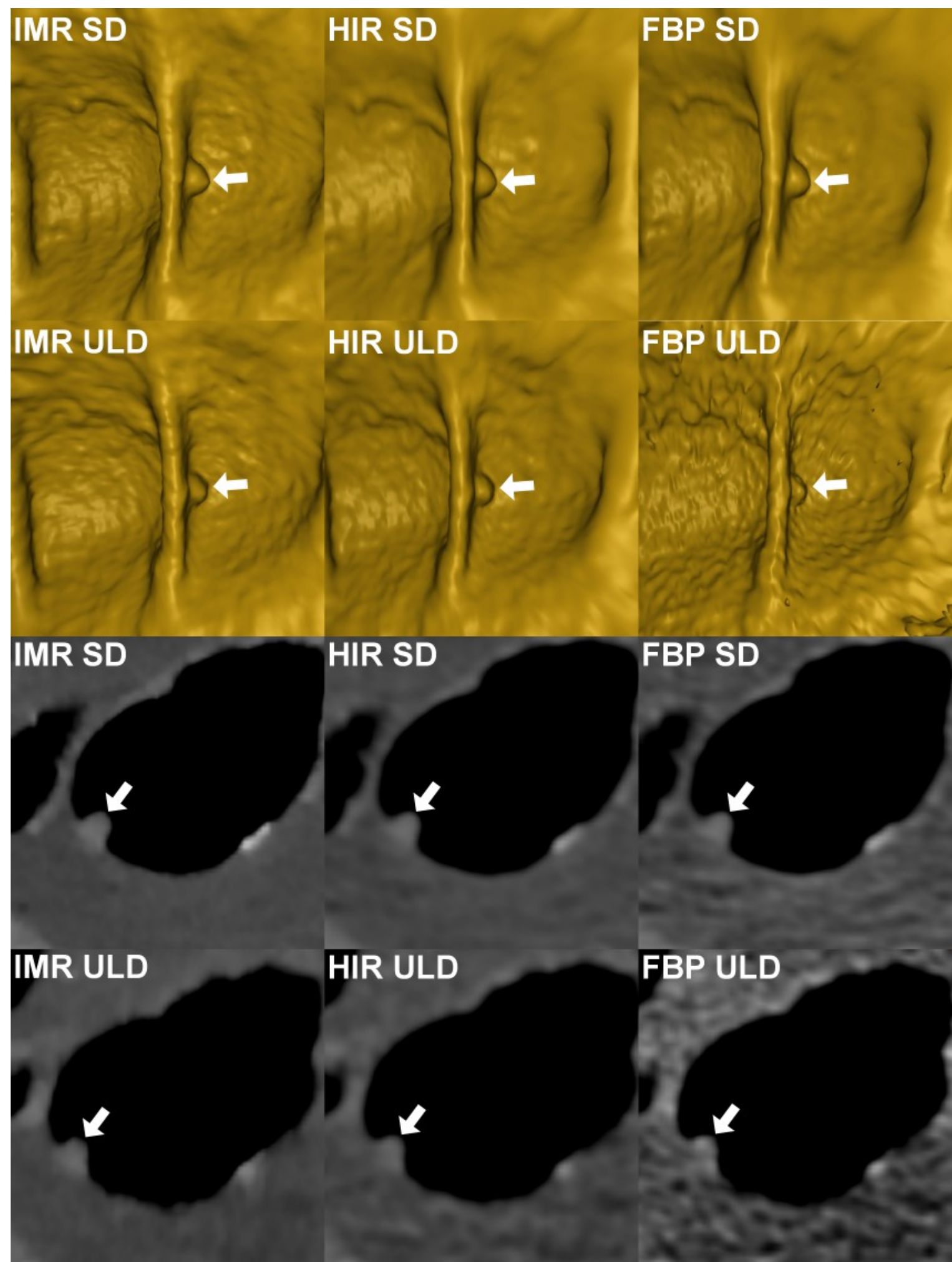

329 Fig. 2. Comparison of image quality of virtual endoscopic view and thin $0.9 \mathrm{~mm}$ sections in a 330 colonic window $(900 / 100 \mathrm{HU})$ of a small $(8.2 \mathrm{~mm})$ sessile polyp (arrow) in cecum shows 331 markedly reduced image quality in ultralow-dose acquisition reconstructed with FBP. FBP = 
332 filtered back projection, HIR = hybrid iterative reconstruction, IMR = iterative model

333 reconstruction technique, $\mathrm{SD}=$ standard dose, $\mathrm{ULD}=$ ultralow-dose.

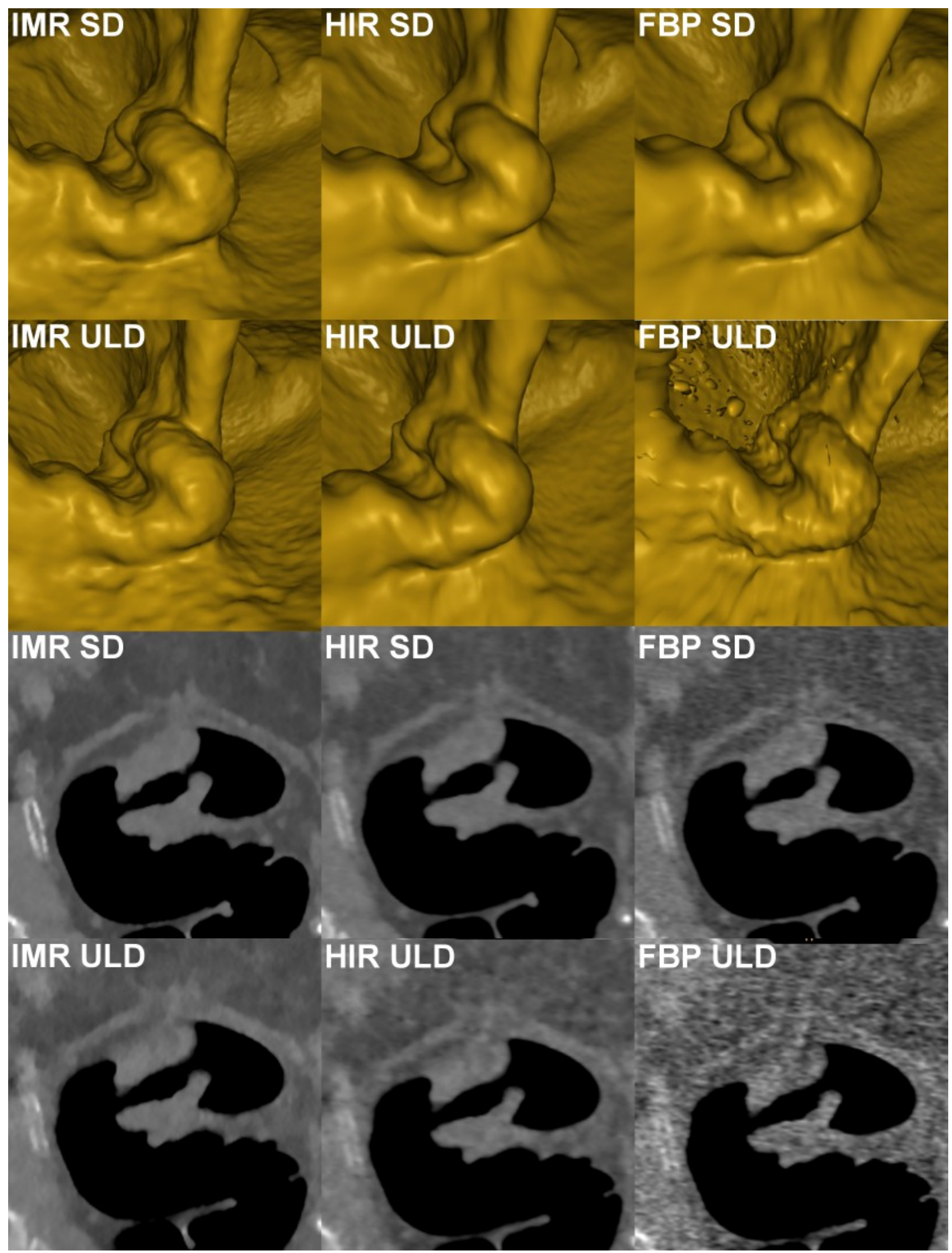

334 Fig. 3. Comparison of image quality of a rectosigmoid tumor in virtual endoscopic view and thin $3350.9 \mathrm{~mm}$ sections in a colonic window $(900 / 100 \mathrm{HU})$ demonstrates markedly reduced image quality 
336 in FBP-ULD. FBP = filtered back projection, HIR = hybrid iterative reconstruction, IMR =

337 iterative model reconstruction technique, $\mathrm{SD}=$ standard dose, $\mathrm{ULD}=$ ultralow-dose.

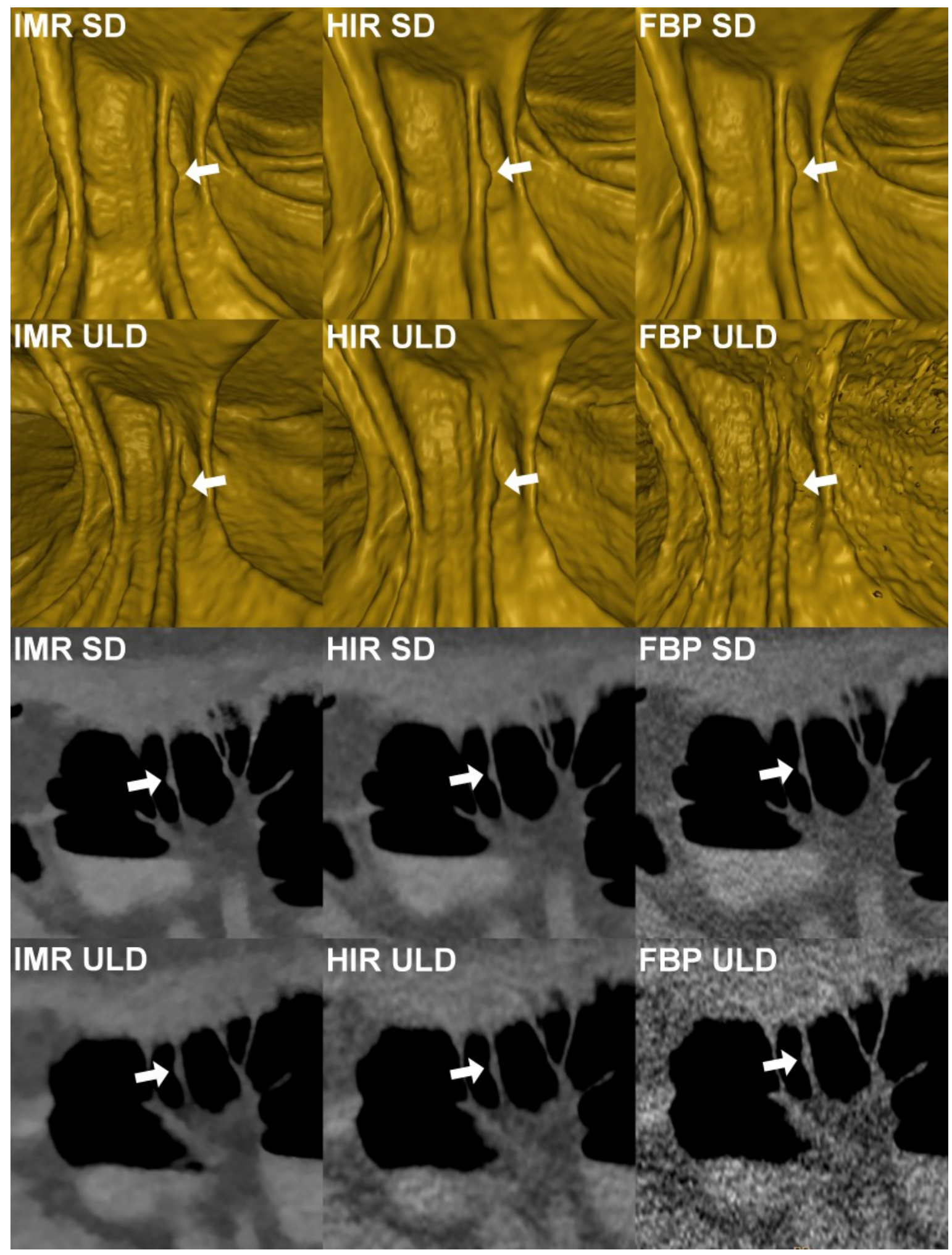

338 Fig. 4. Comparison of image quality of a small $(7.9 \mathrm{~mm})$ flat lesion in the ascending colon shown

339 in virtual endoscopic view and thin $0.9 \mathrm{~mm}$ sections in a colonic window $(900 / 100 \mathrm{HU})$ 
340 demonstrates markedly reduced image quality in FBP-ULD. FBP = filtered back projection, HIR $341=$ hybrid iterative reconstruction, $\mathrm{IMR}=$ iterative model reconstruction technique, $\mathrm{SD}=$ standard 342 dose, $\mathrm{ULD}=$ ultralow-dose.
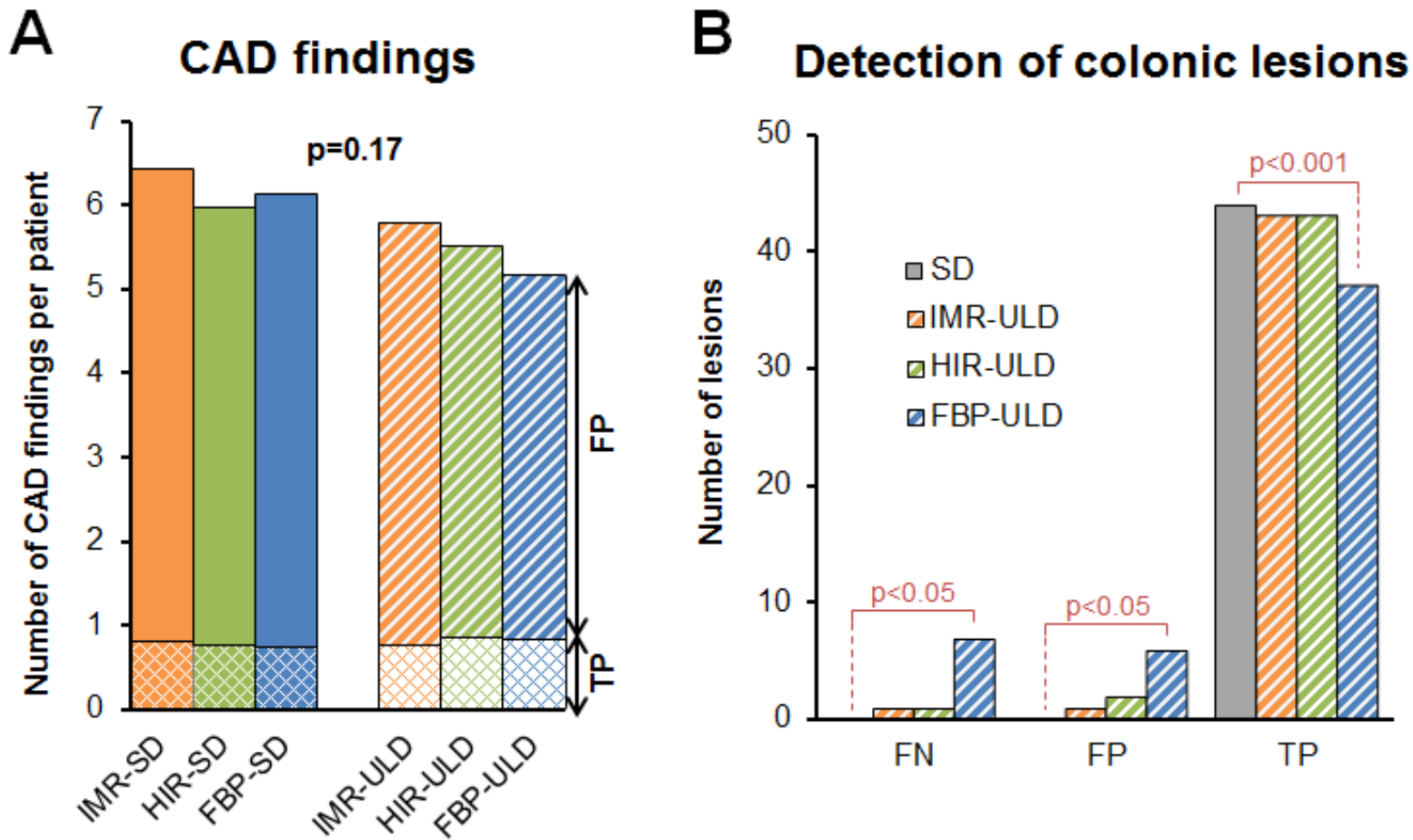

343 Fig. 5. Number of CAD findings represented as cumulative values for supine and prone 344 acquisition (A). True positive (TP, cross-hatched pattern) and false positive CAD marks (FP, 345 diagonal pattern) are distinguished. Detection of colonic lesions in ultralow-dose acquisitions 346 (ULD) compared to standard dose acquisitions (SD) was reduced in FBP, which also had the 347 greatest number of false positive (FP) and false negative (FN) findings (B). FBP = filtered back 348 projection, $\mathrm{HIR}=$ hybrid iterative reconstruction, $\mathrm{IMR}=$ iterative model reconstruction 349 technique, $\mathrm{SD}=$ standard dose, $\mathrm{ULD}=$ ultralow-dose, $\mathrm{CAD}=$ computer aided detection. 
A Noise in aorta and left psoas muscle

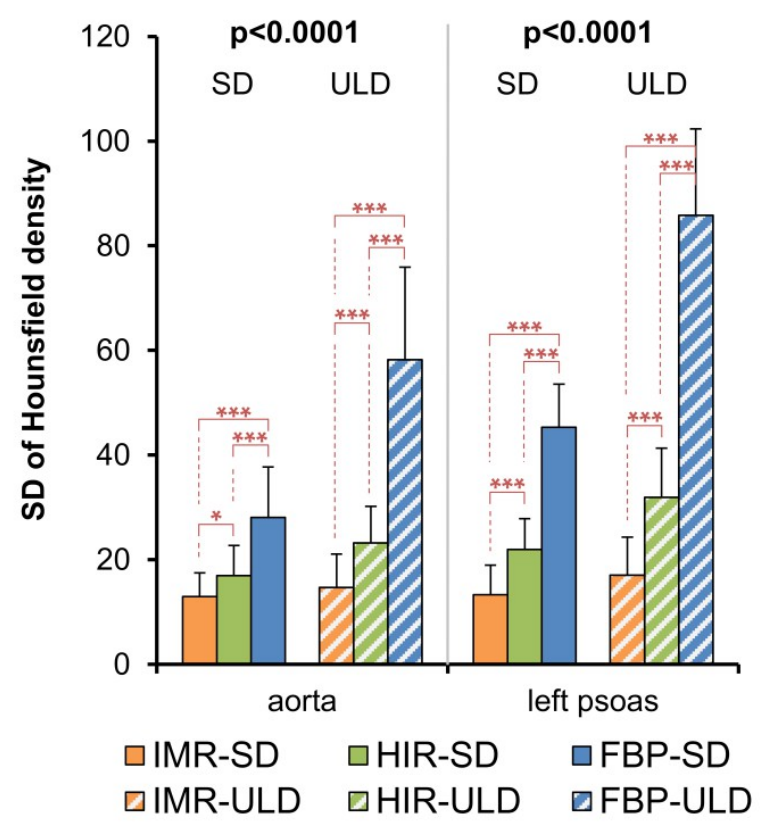

B IQ of left adrenal gland

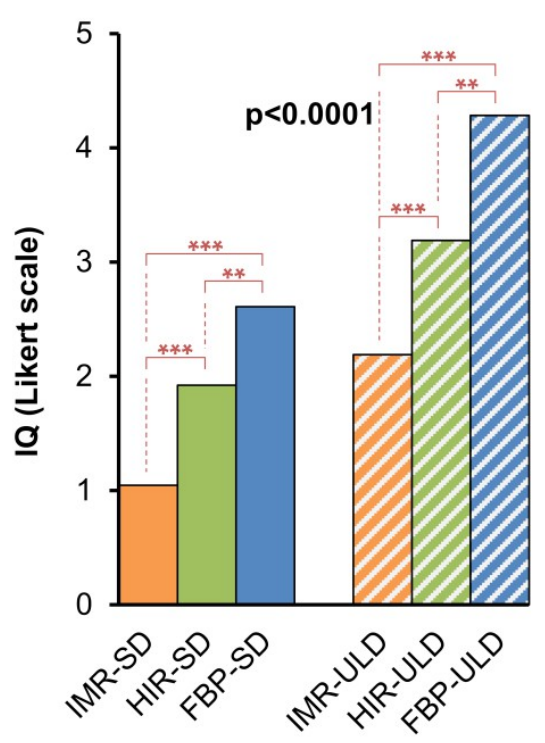

C

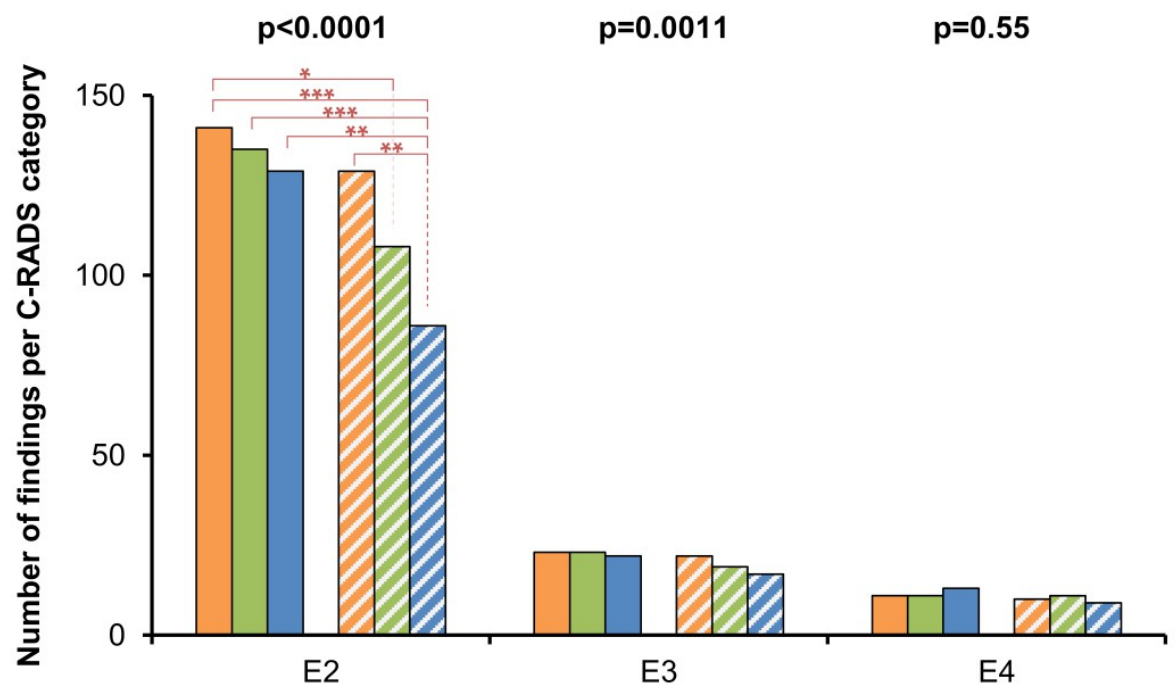

口IMR-SD

$\square$ HIR-SD

$\square$ FBP-SD

$\square$ IMR-ULD

$\square$ HIR-ULD

$\square$ FBP-ULD

350 Fig. 6. Image quality of extracolonic structures represented by image noise in the aorta and the

351 left psoas muscle (A) and rating of image quality (IQ) of the left adrenal gland (B, 1=excellent,

$3525=$ =unevaluable) demonstrate substantial decrease in the image noise especially in ULD with the

353 IMR technique. Diagnostic performance for extracolonic findings grouped by C-RADS

354 classification is reduced in the E2 category (unimportant findings) for low-dose acquisitions 
355 reconstructed with FBP and HIR (C). There is no difference in the E4 category (potentially

356 important findings) and E3 category (likely unimportant findings, incompletely characterized).

$357 \mathrm{FBP}=$ filtered back projection, $\mathrm{HIR}=$ hybrid iterative reconstruction, $\mathrm{IMR}=$ iterative model

358 reconstruction technique, $\mathrm{SD}=$ standard dose, $\mathrm{ULD}=$ ultralow-dose, $*$ : $\mathrm{p}<0.05, * *: \mathrm{p}<0.01, * * *$ :

$359 \mathrm{p}<0.001$. 